\title{
LESÃO MEDULAR POR MERGULHO EM ÁGUAS RASAS: RELATO DE CASO
}

Daniella Augusta Moraes Sanches ${ }^{1}$, Bruna Mariane Nunes Lopes ${ }^{1}$, Gabriel Tiago Viani Silva ${ }^{1}$, Giovanna Neves Bresque ${ }^{1}$, Renata Aparecida de Oliveira Lima ${ }^{2}$, Renata Calciolari Rossi e Silva ${ }^{2}$

${ }^{1}$ Discente e ${ }^{2}$ Docente do Curso de Fisioterapia da UNOESTE, Presidente Prudente, SP.

\section{RESUMO}

O objetivo deste estudo foi avaliar as principais alterações funcionais e motoras antes e após o tratamento fisioterapêutico de um paciente portador de lesão medular decorrente de mergulho em águas rasas. Foi analisado retrospectivamente o prontuário de um paciente portador de lesão medular por mergulho em águas rasas submetido à reabilitação numa clínica de fisioterapia do Oeste Paulista entre os anos de 2008 a 2011, realizando duas sessões semanais de uma hora de duração cada uma. Ao longo do tratamento, o paciente obteve melhora da força muscular sendo observadas as evoluções nas manobras deficitárias. Devido ao tratamento fisioterapêutico o paciente também apresentou melhora da funcionalidade, sendo observadas as evoluções em suas transferências, ou seja, mudanças de posições, entre os anos de 2008 a 2011. Conclui-se que paciente portador de lesão medular apresenta melhora da força muscular e do padrão funcional quando submetido à reabilitação fisioterapêutica.

Palavras-chave: lesão medular, mergulho em águas rasas, fisioterapia, reabilitação, neurologia.

\section{SPINAL CORD INJURY BY DIVING IN SHALLOW WATER: CASE REPORT}

\section{ABSTRACT}

The aim of this study was to evaluate the main motor and functional changes before and after physical therapy of a patient with spinal cord injury caused by diving in shallow water. We analyzed previous records of a patient with spinal cord injury by diving in shallow water, who had rehabilitation at the Rehabilitation Clinical of Oeste Paulista, between 2008 and 2011, attending two one-hour-sessions a week. The patient improved muscle strength as consequence of the rehabilitation program. The treatment also helped the patient improve functionality observing the development while being transferred, in other words, during position changes, between 2008 and 2011. It is understood that patients with spinal cord injury show muscle strength and functional pattern improvement when undergoing physical therapy.

Keywords: spinal cord injury, diving in shallow water, physical therapy, reabilitation, neurology. 


\section{INTRODUÇÃO}

A lesão medular pode ocorrer em diversos níveis da coluna vertebral e por diversas causas, conforme o nível haverá comprometimentos dos movimentos, sensibilidade, controles de esfíncteres, funcionamento dos órgãos, circulação sanguínea e controle de temperatura, pois pode ocorrer uma alteração no sistema nervoso autônomo (PRANDINI; RAMOS, 2004). A lesão ocorre devido à morte dos neurônios e a quebra de comunicação entre os axônios oriundos do cérebro e suas conexões com os neurônios da medula, interrompendo a comunicação entre o cérebro e todas as partes do corpo que ficam abaixo da lesão. As lesões medulares podem ser traumáticas, lesões não traumáticas e congênitas, e ainda completas ou incompletas. As lesões medulares por mergulho em águas rasas são de origem traumática (COELHO et al., 2000).

Acidente por mergulho é uma das principais causas de lesão medular em vários países, representando $19,8 \%$ de todas as lesões medulares cervicais na Polônia; 23,5\% na Rússia; 4\% na Alemanha; 8,5\% nos Estados Unidos; 7\% na Romênia; $11,9 \%$ no Canadá e 14\% na Austrália (GASPAR, 2003).

O local onde ocorreu o acidente talvez seja a única variável de caracterização do acidente por mergulho em águas rasas que difere em função da localidade, país ou região geográfica (CAMPOS et al., 2008).

O predomínio de meio natural, sobretudo dos rios, nos acidentes por mergulho em águas rasas, pode estar diretamente relacionado à formação geográfica nacional (AITO et al., 2005). O Brasil dispõe de uma longa faixa litorânea, paralela a um espaço interior muito grande, banhado por um amplo conjunto de bacias hidrográficas, com muitos rios, de volumes de água variados entre si. O Brasil possui, ainda, um clima predominantemente tropical, marcado por elevadas temperaturas na maior parte do país e em mais da metade do ano. Estes fatores sugerem a existência de um importante potencial de exposição dos indivíduos a locais de mergulho em meio natural, pois são abundantes, atrativos, em função das características climáticas nacionais e, em geral, de acesso irrestrito (SILVA; DELFINO, 2002).

Esse potencial de exposição aos locais de mergulho em meio natural no Brasil amplia-se quando comparado à exposição da população às piscinas. Devido à gratuidade em locais de mergulho comparado às piscinas que, muitas vezes são privadas, limitando o seu uso por classes com menor poder aquisitivo (MURTA; GUIMARÃES, 2007).

Devido à sua biomecânica, a coluna cervical é mais vulnerável ao trauma. Os que sobrevivem às lesões medulares ao nível da coluna cervical são atingidos abaixo da primeira, da segunda e da terceira vértebras (C1, C2 e C3). Isso se deve a dois fatores, o canal vertebral é bastante largo na junção crânio-vertebral, com a medula ocupando apenas 50\% do espaço, o que possibilita, no trauma, que os ossos fragmentados ocupem os espaços vazios, sem atingir a medula (BARSS et al., 2008). Por outro lado, quando a medula é atingida nesses níveis, a chance de sobrevivência é reduzida, pois as lesões completas à altura dessas vértebras interrompem a enervação do músculo diafragma pelo nervo frênico. A ausência de socorro imediato inviabiliza a chance de sobrevivência, devido à falência respiratória imediata (VLOK et al., 2010). As forças que causam lesão na coluna cervical são resultantes de flexões violentas no pescoço. A biomecânica da lesão medular por mergulho em águas rasas combina compressão e hiperflexão 
da coluna cervical. A cabeça do indivíduo, ao atingir o chão da piscina ou o fundo do leito de um rio, recebe o peso do corpo, absorvendo o impacto que causa o dano. Segue-se ao impacto da cabeça, a brusca flexão do pescoço, produzindo fratura de vértebra cervical, que resulta em trauma da medula espinhal (BAMPI et al., 2008).

Quase todos os pacientes com lesão medular precisam de tratamento prolongado. Aqueles com transecções completas apresentam sequelas funcionais e psicológicas pelo resto da vida. A fisioterapia e o suporte emocional precoce são importantes nesta fase. A mobilização precoce previne contraturas, diminui o risco de trombose venosa, protege a pele e reforça o moral (HOWARD, 2006). Um plano de conduta abrangente e individualizado é essencial. Todas estas características são mais bem conduzidas em centros de reabilitação experientes que podem fornecer assistência na modificação doméstica, novo treinamento para dirigir e reabilitação vocacional (ANDRADE; GONÇALVES, 2007). Em quase todos os pacientes ocorre depressão após um período inicial. Se a equipe de reabilitação se prontifica logo a fornecer assistência tanto física como emocional, muitos pacientes com lesão de medula espinhal podem voltar a posições competitivas na sociedade (RODRIGUES; HERRARA, 2004).

É demonstrado que as intervenções fisioterapêuticas nos casos de lesão medular proporcionam bons resultados. Vários protocolos de tratamento são executados neste tipo de paciente, pois a incidência deste tipo de lesão é muito grande. Poucos estudos em nosso país são realizados acerca deste assunto. Dessa forma, faz-se necessária a realização deste trabalho para que novas atualizações e abordagens fisioterapêuticas sejam transmitidas e utilizadas em pesquisas futuras (KHAN; LOPES, 2005).
Assim, este estudo teve como objetivo analisar a evolução de um paciente com lesão medular decorrentes de mergulho em águas rasas destacando as principais alterações funcionais e motoras.

\section{MÉTODOS}

Foi analisado retrospectivamente 0 prontuário de um paciente portador de lesão medular por mergulho em águas rasas que compôs o quadro de pacientes submetidos à reabilitação na Clínica de Fisioterapia da Universidade do Oeste Paulista (UNOESTE) entre os anos de 2008 a 2011. Este projeto foi aprovado pelo Comitê de Ética em Pesquisa da UNOESTE (protocolo no 503/2010).

Foram coletados do prontuário do paciente dados relativos à idade, gênero, diagnóstico e evolução anual dos atendimentos realizados na clínica de reabilitação.

Foi incluído neste estudo o paciente que apresentava lesão parcial de medula decorrente de mergulho em águas rasas. Foram excluídos aqueles que possuírem os prontuários incompletos e os referentes à lesão medular por outro fator etiológico. Como trauma direto decorrente de acidente automobilístico.

Foram analisadas as alterações motoras decorrentes de força muscular e funcionalidade.

As manobras referentes à avaliação da força muscular foram: Queda dos braços estendidos - Paciente, na posição sentada ou de pé, deve manter seus membros superiores na posição de juramento, com os dedos afastados uns dos outros. Depois de alguns segundos ou de meio a um minuto, segundo o grau de déficit motor, o membro parético apresenta oscilações e tende a abaixar-se lenta e progressivamente. Esta prova é útil tanto na avaliação da musculatura proximal como distal dos membros superiores. Mingazzini - Paciente em decúbito dorsal flexiona as pernas em ângulo reto sobre as 
coxas e estas sobre o quadril. Em caso de déficit esta posição não se mantém por muito tempo, surgindo oscilações e/ ou queda progressiva da perna (déficit do quadríceps), da coxa (déficit do psoas) ou de ambos os seguimentos (déficit do quadríceps e do psoas). Barré - Paciente em decúbito ventral, com as pernas flexionadas formando ângulo reto com as coxas. Em caso de déficit surgem oscilações e/ou queda, imediata ou progressiva, de uma ou ambas as pernas. Esta manobra serve para evidenciar déficit dos músculos flexores da perna sobre a coxa (isquiotibiais). Queda dos membros inferiores em abdução - Paciente em decúbito dorsal as pernas são flexionadas sobre a coxa, mantendo-se em apoio plantar bilateral sobre o leito, de tal sorte que os membros inferiores formem com o tronco um ângulo reto. Em caso de déficit motor em um dos lados, surge queda do membro em abdução, de maneira progressiva e imediata (SANVITO, 1998).

As transferências referentes à funcionalidade foram descritas da seguinte forma: Supino para lado, supino para prono, prono para supino, supino para sentado, sentado para quatro, quatro para ajoelhado, ajoelhado para semi-ajoelhado, semi-ajoelhado para em pé.

\section{RESULTADOS}

Este estudo constou com um paciente do gênero masculino, com 21 anos de idade, portador de lesão medular por mergulho em águas rasas, que foi socorrido e encaminhado para o hospital, cuja equipe médica diagnosticou fratura da quinta vértebra cervical e lesão parcial da medula espinhal. Foi realizada a cirurgia para descompressão medular, não tendo recebido atendimento fisioterapêutico no pós-operatório. Após 60 dias do procedimento cirúrgico, foi iniciada a fisioterapia que revelou na primeira avaliação no ano de 2008 que o paciente não adotou posição em nenhuma das manobras realizadas, ou seja, não realizou as manobras referentes à força muscular. A base do tratamento fisioterapêutico deste paciente foi a cinesioterapia com o objetivo de melhora motora e funcional.

Um ano depois de iniciada a fisioterapia (2009) o paciente apresentou melhora da força muscular, representada pela manobra de queda dos braços estendidos cujo resultado apresentouse normal. Em relação à manobra de queda dos membros inferiores em abdução obteve déficit grave, e não adotou posição no restante das manobras.

$\mathrm{Na}$ reavaliação feita no ano de 2010, foram observadas as seguintes evoluções: na avaliação referente à força muscular a manobra de queda dos braços estendidos foi normal, porém, apresentou regressão, não adotando as outras posições exigidas nas manobras seguintes.

$\mathrm{Na}$ última reavaliação (2011) observouse, quanto à força muscular, que o paciente obteve melhora passando para déficit moderado na manobra de queda dos membros inferiores em abdução, mantendo normal o resultado da manobra de queda dos braços estendidos e não adotando posição somente nas demais manobras.

$\mathrm{Na}$ avaliação da funcionalidade 0 paciente apresentou os seguintes resultados: sem dissociação (supino lado, supino prono, prono supino, supino sentado). Acentua padrão de membro inferior (supino lado, supino prono, prono supino, supino sentado). Realiza sem ajuda (supino lado, supino prono, prono supino, supino sentado) e não adota posição (sentado quatro, semi-ajoelhado em pé) (Tabela 1). 
Tabela 1. Descrição das evoluções quanto à funcionalidade realizada em paciente lesado medular por mergulho em águas rasas no ano de 2008.

\begin{tabular}{cc}
\hline Sem dissociação, Acentua padrão de membro inferior, Realiza sem ajuda \\
\hline Supino lado & Prono supino \\
Supino prono & Supino sentado \\
\hline & Não adota posição \\
\hline Sentado quatro & Ajoelhado semi-ajoelhado \\
Quatro ajoelhado & Semi-ajoelhado em pé \\
\hline
\end{tabular}

Na reavaliação realizada em 2009 , obteve melhora nos itens: sem dissociação (supino lado, supino prono, prono supino, supino sentado, sentado quatro). Acentua padrão de membro superior (supino lado, supino prono, prono supino, supino sentado, sentado quatro, quatro ajoelhado). Necessita de ajuda (quatro ajoelhado). Não adota posição (ajoelhado semiajoelhado, semi-ajoelhado em pé). Apresenta retificação endireitamento equilíbrio e proteção REEP (supino lado, supino prono, prono supino, supino sentado, sentado quatro) (Tabela 2).

Tabela 2. Descrição das evoluções quanto à funcionalidade realizada em paciente lesado medular por mergulho em águas rasas no ano de 2009.

\begin{tabular}{cc}
\hline \multicolumn{2}{c}{ Sem dissociação e Apresenta REEP } \\
\hline $\begin{array}{c}\text { Supino lado } \\
\text { Supino prono } \\
\text { Sentado quatro }\end{array}$ & $\begin{array}{c}\text { Prono supino } \\
\text { Supino sentado }\end{array}$ \\
\hline \multicolumn{2}{c}{ Acentua padrão de membro superior } \\
\hline $\begin{array}{cc}\text { Supino lado } \\
\text { Supino prono }\end{array}$ & $\begin{array}{l}\text { Supino sentado } \\
\text { Prono supino }\end{array}$ \\
$\begin{array}{c}\text { Quatro ajoelhado quatro } \\
\text { (necessita de ajuda para realizar) }\end{array}$ \\
\hline \multicolumn{2}{c}{ Não adota posição } \\
\hline Ajoelhado semi-ajoelhado & Semi-ajoelhado em pé \\
\hline
\end{tabular}

Após 12 meses da última avaliação (2010), foram observados os seguintes resultados: Sem dissociação (supino lado, prono supino, supino prono, supino sentado, sentado quatro). Apóia o lado acometido (supino sentado, sentado quatro). Realiza sem ajuda (supino lado, supino prono, prono supino, supino sentado, sentado quatro). Apresenta REEP (supino lado, supino prono, prono supino, supino sentado e sentado quatro). Não adota posição (ajoelhado semi-ajoelhado e semi-ajoelhado em pé) (Tabela $3)$.

$\mathrm{Na}$ última reavaliação realizada no ano de 2011 a evolução constava dos seguintes dados: Sem dissociação (supino lado, supino prono, prono supino). Com dissociação (supino sentado, sentado quatro e quatro ajoelhado). Não adota posição (ajoelhado semi-ajoelhado e semiajoelhado em pé) (Tabela 4). 
Tabela 3. Descrição das evoluções quanto à funcionalidade realizada em paciente lesado medular por mergulho em águas rasas no ano de 2010.

\begin{tabular}{|c|c|}
\hline \multicolumn{2}{|c|}{ Sem dissociação e Apresenta REEP } \\
\hline Supino lado & Prono supino \\
\hline Supino prono & Supino sentado \\
\hline Sentado quatro & \\
\hline \multicolumn{2}{|c|}{ Apóia o lado acometido } \\
\hline Supino sentado & Sentado quatro \\
\hline \multicolumn{2}{|c|}{ Realiza sem ajuda } \\
\hline Supino lado & Prono supino \\
\hline Supino prono & Supino sentado \\
\hline Sentado quatro & \\
\hline
\end{tabular}

Tabela 4. Descrição das evoluções quanto à funcionalidade realizada em paciente lesado medular por mergulho em águas rasas no ano de 2011.

\begin{tabular}{cc}
\hline \multicolumn{2}{c}{ Sem dissociação } \\
\hline \multicolumn{2}{c}{ Supino lado } \\
Supino prono \\
\hline \multicolumn{2}{c}{ Com dissociação supino } \\
\hline $\begin{array}{c}\text { Supino lado } \\
\text { Sentado quatro }\end{array}$ \\
\hline
\end{tabular}

\section{Discussão}

Os resultados desse estudo demonstram que o paciente portador de lesão medular por mergulho em águas rasas obteve melhora da força muscular nas avaliações de 2008 a 2011, sendo que, na manobra de queda dos braços estendidos, passou de não adota posição em 2008 para normal em 2009 a 2011, e queda dos membros inferiores em abdução, passou de não adota posição em 2008 para déficit moderado em 2011. Devido ao tratamento fisioterapêutico o paciente também apresentou melhora da funcionalidade, sendo observadas as evoluções em suas transferências desde os anos de 2008 a 2011.

O traumatismo da coluna vertebral pode lesar de maneira irreversível a medula e suas raízes nervosas (MACHADO, 2002). As lesões traumáticas da coluna e medula espinhal resultam em graus de comprometimento temporário ou permanente das funções neurológicas. A lesão medular pode causar perda parcial ou total da motricidade e sensibilidade, além do comprometimento vasomotor, intestinal, vesical e sexual (PRANDINI; RAMOS, 2004). Estas lesões podem ser divididas em traumáticas, não traumáticas e congênitas, sendo as lesões medulares por mergulho em águas rasas de origem traumática (COELHO et al., 2000).

É demonstrado no estudo de Nunciato et al. (2009) que o paciente estudado, em oito semanas de treinamento muscular, obteve ganho de força muscular no teste de 1 repetição máxima (RM) comparados com os valores de carga máxima vistos antes do treinamento muscular.

A reabilitação dos pacientes inicia-se na fase aguda, sendo um processo que busca o desenvolvimento das capacidades para o paciente poder resgatar sua independência funcional. Isso se encontra no presente estudo, no qual o paciente em questão também obteve ganho de força muscular representado pelas manobras de força muscular, realizadas nas reavaliações após ser submetido ao tratamento fisioterapêutico.

Em estudo realizado com 22 pacientes com lesão medular foi observada melhora na funcionalidade quando o paciente foi submetido a 
um programa de reabilitação comprovando que quando há melhora na funcionalidade desses indivíduos, há também, por consequência melhora na qualidade de vida (RABEH; CALIRI, 2010). Estes dados estão de acordo com os dados encontrados neste estudo o que demonstra a melhora do padrão funcional quando submetidos à reabilitação fisioterapêutica.

Os resultados encontrados neste estudo são ilustrativos do considerável potencial de recuperação associado às lesões medulares incompletas, com ênfase para a correlação significativa entre as melhorias registradas durante o tratamento inicial e nos três anos que se seguem à lesão, como ocorrido no presente estudo, notando-se melhora da força muscular e melhora da funcionalidade desde o início do tratamento até o presente momento. Estes resultados favoráveis poderão ser atribuídos a uma abordagem médica mais abrangente e à elaboração de programas de reabilitação individualizados e intensivos. No entanto, muitos fatores podem influenciar a evolução neurológica e funcional, o que dificulta a demonstração do impacto preciso do tratamento (ANDRADE; GONÇALVES, 2007).

Estudos demonstram que dos pacientes estudados, 16\% apresentavam lesão incompleta ou parcial da medula espinhal (ANDRADE; GONÇALVES, 2007). A maioria dos doentes tetraplégicos apresentava lesão incompleta, assim como o paciente em questão. A evolução mais notória ocorreu nos doentes inicialmente classificados com lesões incompletas ou parciais da medula espinhal, em que 67\% alcançaram função motora preservada abaixo do nível da lesão, o mesmo ocorreu com o paciente deste estudo que na primeira avaliação não apresentava movimentos dos membros superiores, evoluindo no tratamento fisioterapêutico e notando-se melhora observada nas manobras de força muscular, até o presente momento.

Os doentes tetraplégicos alcançaram melhores resultados do que os paraplégicos durante o tratamento. A evolução dos resultados da classificação da escala de avaliação da independência funcional MIF (medida de independência funcional), demonstraram melhoria em $88 \%$ dos doentes. Um ano após a lesão, 54\% dos doentes apresentavam melhorias nos valores obtidos através da MIF, o que está de acordo com nossa pesquisa, no que diz respeito ao paciente estudado também ter obtido melhora da independência funcional após a reabilitação. Não se estabeleceu uma relação significativa entre os resultados funcionais obtidos através da MIF e a gravidade ou o nível de lesão, embora os tetraplégicos tenham alcançado melhores resultados a nível funcional (ANDRADE; GONÇALVES, 2007).

Segundo Vasconcelos e Ribeiro (2011) as informações epidemiológicas obtidas quanto às fraturas da coluna vertebral e lesão medular mostraram que os principais acometidos são homens jovens, em situação de trânsito (carro ou motocicleta). Não houve variação sazonal na incidência e, na maior parte das vezes não houve lesão nervosa. Quando a lesão medular ocorreu, a principal manifestação foi a paraplegia completa. Em nosso estudo observamos dados diferentes em que a causa do traumatismo foi o mergulho em águas rasas e, a principal manifestação foi a tetraplegia parcial. Estes resultados revelam que a lesão medular pode acontecer, também, por outras causas traumáticas.

Em estudo realizado em centro de reabilitação foi destacada a importância da divulgação e realização de campanhas de prevenção do trauma raquimedular para a população brasileira. Assim como a necessidade de encaminhamento precoce destes pacientes ao 
centro de reabilitação, uma vez que a grande maioria é jovem com sequelas neurológicas graves e altamente incapacitantes (CUSTÓDIO et al., 2009). Estes dados corroboram com os nossos, pois o paciente deste estudo é jovem e devido a interevenção fisioterapêutica sua evolução é sentida anualmente.

Conclui-se que este paciente portador de lesão medular apresentou melhora da força muscular e do padrão funcional quando submetido à reabilitação fisioterapêtica durante quatro anos.

\section{REFERÊNCIAS}

Aito S, D'Andrea M, Werhagen L. Spinal cord injuries due to diving accidents. Spinal Cord 2005;43(2):109-116.

DOI:

http://dx.doi.org/10.1038/sj.sc.3101695

Andrade MJ, Gonçalves S. Lesão Medular Traumática- Recuperação Neurológica e Funcional. Acta Médica Portuguesa 2007;2(20):401-406.

Bampi LNS, Guilhem D, Lima DD. Qualidade de vida em pessoas com lesão medular traumática: um estudo com o WHOQOL. Revista Brasileira de Epidemiologia 2008;11(1):67-77. DOI: http://dx.doi.org/10.1590/S1415-

790X2008000100006

Barss P, Djerrari H, Leduc BE, Lepage Y, Dionne CE. Risk factors and prevention for spinal cord injury from diving in swimming pools and natural sites in Quebec, Canada: A 44-year study. Accident Analysis and Prevention 2008;40(2):787797.

DOI:

http://dx.doi.org/10.1016/i.aap.2007.09.017

Campos FM, Ribeiro AT, Listik S, Pereira CAB, Sobrinho JA, Rapoport A. Epidemiologia do traumatismo da coluna vertebral. Revista do Colégio Brasileiro de Cirurgiões 2008;35(2):88-93. DOI: $\quad$ http://dx.doi.org/10.1590/S0100$\underline{69912008000200005}$

Coelho DG, Brasil AVB, Ferreira NP. Fatores de risco de lesão neurológica nas fraturas e luxações da coluna cervical baixa. Arquivo Neuropsiquiátrico 2000;58(4)1030-1034.

Custódio NRO, Carneiro MR, Feres CC, Lima GHS, Jubé MRR, Watanabe LE, Saliba LGRSO, Daher S, Garcia ACF. Lesão medular no Centro de Reabilitação e Readaptação Dr. Henrique
Santillo 2009;8(3):265-268.

Gaspar AP. Avaliação epidemiológica dos pacientes com lesão medular atendidos no Lar Escola São Francisco. Acta Fisiátrica 2003;10(2)73-77.

Howard WM. Coluna vertebral: Terapia manual. 1ed. São Paulo: Guanabara koogan; 2006.

Khan RL, Lopes MHI. Mergulho em águas rasas e lesão medular: uma abordagem educativa e preventiva. Scientia Médica, 2005;15(2):121-124.

Machado ABM. Neuroanatomia funcional. 2ed. São Paulo: Atheneu; 2002.

Murta SG, Guimarâes SS. Enfrentamento à lesão medular traumática. Estudo de Psicologia 2007;12(1):57-63.

Nunciato AC, Pastrelo D, Leite RD, Prestes J, Medalha CC. Treinamento de força e treinamento funcional em adolescente lesado medular-Relato de caso. Conscientiae Saúde 2009;8(2):281-287. DOI:

http://dx.doi.org/10.5585/conssaude.v8i2.1644

Prandini MN, Ramos PS. Avaliação de fatores que influenciam a função respiratória em pacientes portadores de lesão medular completa em níveis cervicais inferiores. Revista Brasileira de Neurologia 2004;40(1):40-47.

Rabeh SAN, Caliri MHL. Capacidade funcional em indivíduos com lesão de medula espinhal. Acta Paulista de Enfermagem 2010;23(3):321327. DOI: http://dx.doi.org/10.1590/S010321002010000300002

Rodrigues D, Herrara G. Recursos fisioterapêuticos na prevenção da perda da densidade mineral óssea com lesão medular. Acta Ortopédica Brasileira 2004;12(3):183-188. DOI: $\quad$ http://dx.doi.org/10.1590/S141378522004000300008

Sanvito WL. Propedêutica neurológica básica. 6ed. São Paulo: Atheneu; 1998.

Silva CLC, Defino HLA. Estudo epidemiológico das fraturas da coluna cervical por mergulho na cidade de Ribeiräo Preto-SP. Medicina 2002;35(1):41-47.

Vasconcelos ECLM, Riberto M. Caracterização clínica e das situações de fratura da coluna vertebral no município de ribeirão preto, propostas para um programa de prevenção do trauma raquimedular. Coluna/Columna 2011;10(1):40-43. 
Vlok AJ, Petersen J, Dunn RN, Stander J.

Shallow-water spinal injuries - devastating but preventable. South Africa Medical Journal 2010;100:682-684. 